Running Head: A method for evaluating PPG-based HRV devices

When is it good to use wristband devices to measure HRV?: Introducing a new method for evaluating the quality of data from photophlethysmography-based HRV devices

\author{
William S. Ryan \\ University of Toronto, St. George \\ James Conigrave \\ Geetanjali Basarkod, \\ Joseph Ciarrochi, \\ \& Baljinder K. Sahdra
}

Institute for Positive Psychology and Education, Australian Catholic University

Author Notes:

Correspondence concerning this article should be addressed to William S. Ryan, Department of Psychology, University of Toronto, St. George, 100 St. George St. Sidney Smith Hall Toronto, ON M5S 3G3. Email: thewillryan@gmail.com. Phone: 647-551-0496.

The authors declare that they have no conflicts of interest. 
Running Head: A method for evaluating PPG-based HRV devices

\begin{abstract}
Objective: Recent technological advances have led to the proliferation of ambulatory devices for non-invasively assessing cardiac activity. While these devices have exciting implications for conducting research outside the laboratory, it is critical that this increased mobility does not compromise data quality. As a test case, we assess the efficacy of Empatica's E4, a high-end wristband device designed to assess Heart Rate Variability (HRV) through the use of photoplethysmography. Approach: We compare the E4 to traditional, wired electrocardiogram measures across a variety of conditions, including seated, supine, and standing baselines, as well as typing and grip strength tasks. Most importantly, we introduce and demonstrate the efficacy of a new method for determining the amount of error in HRV estimates derived from the E4 and a technique for adjusting error tolerance. Main Results: Results indicate that the E4 is severely compromised by motion artifact, resulting in a high percentage of missing data across all conditions except seated and supine baselines. Employing error adjustment yielded more robust results, but at the cost of significantly reducing sample size where motion artifact was present.
\end{abstract} Significance: These results call into question the wristband's efficacy as an HRV measurement tool in most in-vivo conditions. We recommend that researchers interested in using photoplethysmography-based HRV devices use caution and evaluate the data quality using methods for error detection and tolerance, such as the one presented here. Keywords: heart rate variability, ambulatory photoplethysmography, electrocardiogram. 
When is it good to use wristband devices to measure HRV?: Introducing a new method for evaluating the quality of data from photophlethysmography-based HRV devices

Recent technological advances have led to the proliferation of ambulatory devices for non-invasively assessing cardiac activity. Wristband devices, in particular, have become popular for tracking heart rate as well as other fitness-related parameters such as daily activity and calories burnt. The majority of these devices are developed for the consumer market, although some, like Empatica's E4 (http://www.empatica.com), are designed specifically with physiological research in mind, including measurement of heart rate variability (HRV). These wearable devices have important implications for physiological research as they enable data collection outside of the laboratory, allowing for the measurement of physiological activity as it occurs in reaction to real-life situations (e.g Muaremi, Arnrich, \& Tröster, 2013). Wristband devices are also relatively inexpensive and significantly more comfortable than traditional, labbased cardiac measures, facilitating the collection of data from more participants over longer periods of time. There is currently, however, little research examining the validity and reliability of cardiac measures, such as heart rate variability (HRV) derived from wristband devices (McCarthy, Pradhan, Redpath, \& Adler, 2016; Ollander, Godin, Campagne, \& Charbonnier, 2016). The present research seeks begins to fill this gap by introducing a new method of evaluating the quality of data obtained from photoplethysmography (PPG)-based measurements of HRV. As a test case, we compared HRV measures derived from Empatica's E4 (http://www.empatica.com), a wristband device designed specifically for physiological research, to those derived from traditional ECG. We then subjected the data obtained from E4 to our new method of error detection to more closely evaluate the quality of data obtained from E4.

\section{Heart Rate Variability}


Heart rate variability (HRV) is a non-invasive means of assessing autonomic function, with greater variability generally interpreted as indicating greater autonomic control (Berntson et al., 1997; Porges, 2007; Saul, 1990). The variability in HRV refers to oscillations in the interval between consecutive heartbeats or instantaneous estimations of heart rate. These varying distances are referred to as inter-beat intervals (IBIs) and result from the relative activation and/or inhibition of the sympathetic and parasympathetic branches of the autonomic nervous system and their respective influences on the heart (Berntson, Cacioppo, \& Quigley, 1991). Under conditions of stress, sympathetic nervous system activation increases the firing rate of the sino-atrial node, the heart's pacemaker, increasing heart rate (Appelhans \& Luecken, 2006). The parasympathetic nervous system, acting primarily via vagal innervation of the heart, has an opposing, inhibitory effect on sino-atrial node firing, acting as a break to slow down heart rate (Katona \& Jih, 1975; Bertsch, Hagemann, Naumann, Schächinger, \& Schulz, 2012). Heart rate variability results from the co-activation, co-inhibition, or the activation of one and inhibition of the other of the parasympathetic and sympathetic branches (Bernston et al., 1991). These two branches of the ANS operate in concert to adaptively and dynamically regulate cardiac function in response to changing circumstances and external stimuli (Acharya, Joseph, Kannathal, Lim, \& Suri, 2006).

Vagal innervation is impacted by the respiration cycle; it is suppressed on inhalation leading heart rate to fluctuate as the vagal break is turned on and off with exhalation and inhalation, respectively. Variability within the respiration frequency band is termed respiratory sinus arrhythmia (RSA) and is used to index vagal efferent activity, or vagal tone, non-invasively (Grossman \& Taylor, 2007). Greater variability in RSA equates with greater vagal strength or tone (Bertsch, et al., 2012; Grossman \& Taylor, 2007) and is associated with a host of positive 
socioemotional outcomes such as connectedness, positive emotions, interpersonal style, attention, and working memory (e.g. Kok \& Fredrickson, 2010; Thayer, Hansen, Saus-Rose, \& Johnson, 2009).

Due to the non-invasive nature of RSA, or high frequency HRV, it is assessed in a wide variety of domains. Initially, HRV was primarily utilized for its clinical applications, as a means of indexing cardiac health and disease (e.g. Akselrod, et al., 1981; Carney, et al., 2001; Guzzetti, et al., 1988; Kamath \& Fallen, 1995; Pagani, et al., 1986; Masi, Hawkley, Rickett, \& Cacioppo 2007; Thayer \& Lane, 2007). It has also been employed to monitor individuals with diabetes (Ewing, Borsey, Bellavere, \& Clark, 1981), detect renal failure (Akselrod, Eliash, Oz, \& Cohen, 1987), track physical fitness (Davy, DeSouza, Jones, \& Seals, 1998), diagnose sleep disorders (Drinnan, Allen, Langley, \& Murray, 2000), track sleep quality (Elsenbruch, Harnish, \& Orr, 1999), and assess the impact of tobacco smoke (Pope, et al., 2001) and alcohol (Malpas, Whiteside, \& Maling, 1991) on cardiac function. For a review of physiological applications of HRV, see Acharya, et al. (2006).

More recently, HRV has also been used to assess a growing number of psychophysiological processes and outcomes related to cardiovagal control, including attention (Porges, 1992), emotion regulation (Calkins \& Johnson, 1998), self-regulation (Geisler, Kubiak, Siewert, \& Weber, 2013), stress reactivity (Fabes \& Eisenberg, 1997), anxiety (Friedman, 2007), depression (Chambers \& Allen, 2002; Rottenberg, 2007), group affiliation (Sahdra, Ciarrochi \& Parker, 2015), and wise reasoning (Grossmann, Sahdra \& Ciarrochi, 2016), among others. HRV has been examined as both a trait-like individual difference variable and a state-like variable that fluctuates within and between persons in relation to specific circumstances, tasks, or interventions (e.g. Miu, Helman, \& Miclea, 2009). The popularity of HRV measures is due 
largely to the fact that they are relatively easy to collect, non-invasive, and reproducible (Ge, Srinivasan, \& Krishnan, 2002; Kleiger, et al., 1991).

\section{Metrics of Heart Rate Variability}

Thus far, we have discussed HRV as if it were a unified construct. However, there are multiple methods for calculating HRV from a given cardiac signal that reflect different components of autonomic control over the cardiovascular system. Most common in psychophysiological research are methods that operate in the time domain and those that operate in the frequency domain (for comprehensive review of all HRV calculation methods see Allen, Chambers, \& Towers, 2007).

Time domain measures. These examine the intervals between each QRS complex in the

ECG wave directly. These intervals are calculated based on the time between successive R-peaks and are referred to as RR intervals, inter-beat intervals (IBIs), or normal-to-normal (NN) intervals (see Figure 1). Statistical parameters can then be calculated using the NN intervals as input data. Assessed in milliseconds, these time-based measures are the simplest to calculate and at their most basic include mean heart rate and mean NN interval (Stein \& Kleiger, 1999). Additional parameters that reflect the variation in IBIs may also be calculated from the NN time series data itself. For example, SDNN, one of the most common time-based measures of HRV, is simply the standard deviation of the NN intervals (Murray et al., 1975). SDNN estimates overall HRV and reflects both short and long-term variation in the timing of cardiac cycles when measured over a long period of time. Critically, SDNN values are heavily dependent on the length of the recording and so must be interpreted with caution when comparing across studies with different designs (Ewing et al., 1981; Saul, Albrecht, Berger, \& Cohen, 1987). It is, therefore, recommended that recordings be standardized to 5 minutes in length (Task Force of 
the European Society of Cardiology and the North American Society of Pacing and Electrophysiology, 1996). To estimate just the long-term components, the standard deviation of the average $\mathrm{NN}$ intervals calculated over 5-minute periods of time (SDANN) may be used (Acharya, et al., 2006).

Statistical parameters can also be calculated based on the differences between adjacent intervals. These include the standard deviation of successive differences between NN intervals (SDSD) and the root mean square of successive difference intervals (RMSSD; Acharya, et al., 2006, Allen, et al., 2007). Of these difference-based measures, RMSSD is recommended for its statistical properties and because it reliably estimates short-term variation in the timing of cardiac cycles (Task Force, 1996).

Spectral Analysis. Heart rate variability can also be examined within specific frequency ranges, allowing the researcher to home in on specific influences on the variability, such as respiration. Here the IBI series is subjected to a Fourier transform or autoregressive modeling to represent it in the frequency domain. This method is often termed spectral analysis as it yields a power spectrum, the specific frequency bands of which can then be examined. Activity in the low frequency range $(0.04-0.12 \mathrm{~Hz})$ is associated with activity of the baroreflexes (Goldstein, Bentho, Park, \& Sharabi, 2011) and is posited by some researchers to index sympathetic tone especially when measured over long periods of time (Pagani et al., 1997). Fluctuations in the high frequency range $(0.12-0.4 \mathrm{~Hz})$ reflect the impact of parasympathetic nervous system activity on heart rate via the vagus nerve (Berntson, et al., 1997; Akselrod, et al., 1981). Power in this range is believed to be indicative of a relatively stable autonomic disposition (Bertsch et al., 2012; Thayer et al., 2009) and has been linked with a host of positive traits and outcomes related to socio-cognitive functioning (e.g. Appelhans \& Luecken, 2006; Thayer et al., 2009). 
Examining the full frequency range, or total power, provides an estimate of overall variability and overall autonomic activity and is highly correlated with SDNN (Task Force, 1996).

\section{Collecting Heart Rate Variability Data}

Electrocardiogram. HRV is traditionally assessed using an electrocardiogram (ECG), which records the electrical activity of the heart using bipolar sensors placed along Einthoven's triangle (Einthoven, Fahr, \& De Waart, 1913). The resulting ECG waveform reflects changes in voltage associated with the various phases of the cardiac cycle. Specifically, the QRS complex indicates ventricular depolarization and its peaked shape makes it a robust and easily identified index of cardiac chronotropy. The distance between the R peaks in milliseconds form the interbeat interval (IBI) series that is used to calculate the majority of HRV metrics (see Figure 1).

Traditionally, ECGs have been rather cumbersome hospital or lab-bound devices. While these are suitable for many types of clinical and other research, being tethered restricts options for monitoring people long-term and conducting longitudinal and ecologically valid research. For this reason, ECGs themselves have become increasingly ambulatory (Sandercock, Shelton, Bromley, \& Brodie, 2004; Koudstaal, van Gijn, Klootwijk, vad der Meche, \& Kappelle, 1986; Jaboudon, Sztajzel, Sievert, Landis, \& Sztajzel, 2004), with some applications integrating ECG sensors into textiles in the form of sensorized t-shirts (Paradiso, Faetti, \& Werner, 2011). Despite their designation as ambulatory devices, many of these wearable ECGs are highly sensitive to motion artifact (Romero, et al., 2011), leading many to recommend data collection during sleep (e.g., Muaremi, et al., 2013) and driving research into motion correction algorithms (e.g. Alqaraawi, Alwosheel, \& Alasaad, 2016).

Photoplethysmography. Interest in the ambulatory measurement of cardiovascular activity and autonomic function has driven research into alternative assessment methods. One of 
the most promising of these new methods is the estimation of cardiac chronotropy using pulse signals or photoplethysmography (PPG) (e.g., Schäfer \& Vagedes, 2013; Bolanos, Nazaran, \& Haltiwanger, 2006). PPG methods detect blood volume pulse (BPV) or variation in the volume of atrial blood in the microvasculature of peripheral tissues resulting from the cardiac cycle (Challoner, 1979). This is accomplished by shining a small LED light on the wrist, fingertip, earlobe, or forehead and then measuring how much of that light is either absorbed or reflected (depending on whether transmission or reflection PPG is being done). When blood volume is greater, more light is absorbed, meaning that there is less light available to be detected by the PPG sensor. In this way, changes in blood volume can be tracked to reflect the number and timing of cardiac cycles (Kamal, Harness, Irving, \& Mearns, 1989), allowing for the calculation of pulse rate and pulse rate variability (PRV). The peaks of the PPG wave can be used just like the R-peaks of the ECG waveform to estimate the number of heartbeats as well as the intervals between them (IBIs). The primary difference between the two types of measurement is pulse transit time (PTT), or the time it takes for the pulse wave to travel from the heart to the periphery (Jago \& Murray, 1988). Thus, PRV and HRV measures should yield highly similar results. Indeed, research indicates that the two measures are highly correlated, especially when assessed at rest among healthy participants (Foo \& Wilson, 2006; Schäfer \& Vagedes, 2013; Lu, Yang, Taylor, \& Stein, 2009; Rauh, Limley, Bauer, Radespiel-Troger, \& Mueck-Weymann, 2004). For example, using a finger pulse oximeter to assess PPG alongside a traditional ECG, Jeyhani, Mahdiani, Peltokangas, and Vehkaoja (2015) found PPG- and ECG- derived HRV metrics to be highly correlated with one another, with the exception of the pNN50 metric.

Importantly, the majority of these studies have examined correspondence between the two methods under relatively motion-free conditions. Indeed, Gil and colleagues (Gil, et al., 
2010) were among the first to test the efficacy of PPG-derived HRV under non-stationary conditions. While these authors did find good correspondence between the two methods, the non-stationary condition assessed was the tilt-table test in which the participant moves very little volitionally, but rather, is positioned at different degrees of inclination by a moving table (Julu, Cooper, Hansen, \& Hainsworth, 2003). Lu and Yang (2009) conducted a similar comparison of traditional ECG and PPG measured at the fingertip. They found that when high quality PPG data were obtained, HRV values from the two methods were highly correlated. However, data were not always usable; motion artifact prevented analysis of PPG data from a significant number of participants. While these results may appear somewhat surprising given that participants were instructed to sit still for the duration of each 5-minute recording epoch, this finding is consistent with other researchers' concerns regarding the high sensitivity of PPG to motion artifact (Allen, 2007).

In part because of problems with motion artifact and partly in an effort to maximize comfort when worn long-term, there has been increasing research into measuring PPG using wristband devices (Garbarino, Lai, Bender, Picard, \& Tognetti, 2014; Tamura, Maeda, Sekine, \& Yoshida, 2014). Arberet and colleagues (2013) compared HRV measured by a wrist PPG sensor (CSEM propriety wrist monitor) to traditional ECG-derived HRV assessed overnight and found the two measures to correlate at approximately .90 for both temporal and spectral HRV metrics. While high, these correlations were based on data obtained during sleep when presumably participants were moving very little, almost certainly much less than they would move during the day.

Renevey, Vetter, Krauss, Celka, and Depeursinge (2001) developed and tested a similar wrist-based PPG device with an accelerometer. Using their motion correction algorithms, they 
found acceptable rates of error during both baseline and physical activity (running). However, data were collected only over 10-second intervals. Preejith, Annamol, Jayaraj, \& Mohanasankar (2016) tested another, similar wristband device and also found a high degree of correlation with traditional ECG. This study, however, was conducted in a hospital setting and data were collected for only 20 seconds at a time. Still, motion artifact was noted as an issue.

In this climate of growing interest in wrist-worn PPG devices for HRV measurement, many devices have been introduced in the market. Consider, for example, the Empatica E4, the latest model of Empatica wristbands. The E4 developed by Empatica (www.empatica.com) is designed for use by researchers interested in tracking these measures over time and outside of the laboratory. The device is somewhat similar in appearance to the Fitbit, Apple watch, and other commercially available health tracking devices worn on the wrist. However, it is notably larger than these commercial models and does not have a display screen. A primary difference between the E4 and these more commonly used devices is that, with the E4, it is possible for the researcher or user to gain access to the full time-series of data rather than only summary statistics. Although not discussed in this paper, the E4 can also assess electrodermal activity (EDA) by applying a very low $(8 \mathrm{~Hz})$ alternating current to the inside of the wrist and assessing resistance. In addition, the $\mathrm{E} 4$ also contains an accelerometer that detects motion along 3 axes $(\mathrm{X}, \mathrm{Y}, \mathrm{Z})$.

The E4 is marketed specifically to researchers as a "wearable wireless device designed for continuous, real-time data acquisition in daily life" for assessing HRV under various conditions in and outside of the laboratory (www.Empatica.com). However, for researchers interested in using the E4 for its advertised purpose, there is a dearth of peer-reviewed studies demonstrating its validity as a turnkey tool for continuous measurement of HRV in different 
contexts. Empatica's previous model, the E3, was touted as an improvement on finger-based measures, especially during tasks employing minimal wrist motion such as typing (Garbarino, et al., 2014), and the E4, presumably, is an improvement on the E3. Like other commercially available wristband devices, the E3 and the E4 utilize an accelerometer paired with proprietary algorithms for detecting motion and removing data contaminated by motion artifact (Garbarino, et al., 2014).

As far as we know, there are only three peer-reviewed published studies in which researchers attempted to evaluate E4's performance using different benchmarks. In one study by McCarthy and colleagues (2016), participants wore the E4 and a Holter ambulatory ECG monitor for 24 to 48 hours. A subset of 15-second overlapping segments of data were sampled. The researchers indicated that over $85 \%$ of these segments contained usable data from both devices. However, of the $15 \%$ of cases in which one device yielded poor quality data, this was twice as likely to be due to failure of the PPG rather than the ECG. Importantly, the majority of good quality data were collected during the night, presumably when motion was minimal.

In another attempt to test E4's validity in a controlled lab context, Ollander and colleagues (2016) compared the E4 with classic ECG and finger conductivity electrodes during a social stress task. They found that the E4 failed to detect a significant number of inter-beat intervals, while time-domain features (i.e. heart rate) were accurately estimated. However, as only seven participants (one male) were included in this study, more work is needed to establish the reliability and generalizability of these results. Similarly, Corino, Matteucci, and Mainardi (2007) examined the E4's performance in a highly controlled context. They analyzed BVP signals from the E4 to develop algorithms for detecting atrial defibrillation. Recordings were obtained at rest but only 2 minutes of a 10-minute segment were analyzed. Critically, this study 
did not assess the reliability of the E4 during daily activities that include motion. While promising, these preliminary studies on the E4 do not show a systematic comparison of the E4's performance across different conditions, as compared to the performance of the gold-standard classic ECG-based measurement of HRV in the same conditions.

\section{Present Research}

The purpose of this study is to devise a method for evaluating the quality of HR and HRV data obtained using a purportedly research-grade wristband device that utilizes reflective PPG to monitor cardiac chronotropy. We employ Empatica's latest model, E4 as test case, but our method is general enough to be applied to similar data obtained by other wrist-band devices if the raw data is accessible to researchers. The E4 has strong promise as a tool for researchers wishing to assess cardiovascular functioning with minimal, non-invasive equipment and outside the laboratory. However, although research has been done to confirm the safety and validity of these devices (McCarthy et al., 2016; Ollander, et al., 2016), little has been done to validate derived measures across a variety of contexts (including those that require movement) and in large samples.

The goal of the present research is to conduct such validation tests using traditional methods of correlating the data obtained from the test device with simultaneous data obtained from a gold-standard device. Additionally, we also aim to test our new method for a more finegrained evaluation of the quality of data obtained from these devices. Specifically, we first compare heart rate (HR) and heart rate variability (HRV) values derived from the E4 to those obtained from a traditional ECG (Biopac, Goleta, CA), when collecting data simultaneously with both systems. Previous research testing the validity of the E4 has utilized data collected continuously over the course of a day (or more) during which undifferentiated activity has 
occurred, or has assessed HRV in relation to just one specific stress test. The current research improves on these methods by systematically comparing measures derived by the E4 and a traditional, wired ECG system across a variety of conditions. Specifically, participants' HRV was assessed during standing, seated, and supine rest, as well as during computer and grip strength tasks. We selected these conditions to reflect postures and tasks that people are likely to engage in both within and outside the laboratory. This allows us to examine the reliability and validity of the E4 under conditions most likely to be relevant to researchers and to assess the effects of these tasks and postures on motion artifact.

Equally importantly, the present study contributes to the literature on ambulatory HRV measures by offering a new method (as detailed below) for determining the amount of error in HRV estimates and for adjusting error tolerance. This method assesses the percentage of heartbeats the device failed to detect. The researcher can then select a cutoff value, and exclude all cases with missingness greater than this value. Employing these methods yields more robust results than others have obtained using the proprietary algorithms included in the E4 software.

\section{Method}

\section{Participants}

A total of 78 undergraduates from the University of California, Santa Barbara participated in this study. Informed consent was obtained from all participants before commencing the study procedures and all participants were compensated with course credit. Responses to the health screening form indicated that all participants were in good cardiovascular health. Of the 78 total participants, 56 were women, 21 were men, and one identified as genderqueer/non-binary. Participants ranged in age from 18 to $30(M=19.85, S D=$ 
1.77) and were relatively ethnically diverse: $37.2 \%$ White, $20.5 \%$ Asian/Asian American, 20.5\%

Latino/Latina, 10.3\% Multi-racial, 3.8\% Black/African American, 3.8\% Arabic/Middle Eastern, and $3.8 \%$ selected "other".

\section{Procedures}

Upon arriving at the lab, a trained research assistant described the study procedures and obtained informed consent from the participant. The research assistant then applied the electrodes and wristband device (E4) necessary for the collection of relevant physiological signals (see Measures \& Materials section below). The participant then performed a 6-minute seated baseline followed by two additional 3-minute baseline periods, one standing and one supine (the order of the latter two baselines was counter-balanced). Next, participants completed a computer-based survey assessing demographic information, well-being, and other psychosocial variables. Given our goal of comparing physiological indices, we do not report on these psychosocial variables in the present research. However, we do utilize the physiological data collected while participants were completing these measures to assess the impact of the motion of using a mouse and typing on resultant data. Participants then completed a hand-grip strength task where they were instructed to squeeze a digital dynamometer as hard as possible three times, each trial separated by approximately 10 seconds and at the signal of the experimenter.

Participants did this for both their dominant and non-dominant hands (order counter-balanced). After this, the research assistant removed all sensors from the participant. Finally, the participant was debriefed as to the purpose of the study, thanked, and dismissed.

\section{Physiological Measures}

Electrocardiograph (ECG). ECG signals were recorded continuously using a Biopac MP150 system with ECG amplifier (Model ECG 100C) and a modified lead II electrode 
configuration (Sherwood, et al., 1990). Data were integrated with a Biopac MP150 and stored using Acknowledge software (version 4.3, Goleta, CA).

E4 wristband. The E4 wristband from Empatica was used simultaneously to assess PPGderived HRV. This device works by simply placing the watch-like wristband around either the left or right wrist. For all participants it was placed on the dominant hand for the duration of the study, except for when moved to the non-dominant wrist when testing grip strength of the dominant hand (to reduce motion artifact). Data collected using the E4 was uploaded to Empatica Connect, a secure site maintained by Empatica where the researcher can visualize and download the data stream. Data were stored and identified using only a numeric string reflecting the date and time when the data were collected.

Once data collection for all participants was complete, all data were downloaded from the Empatica website as a series of zip folders. Using the statistical program, R (R Core Team, 2017), each .csv containing IBIs were combined into a single data frame. Using an experiment $\log$ that noted the timing of each participant's data collection, this data frame was then filtered to create windows of analysis for each participant and each task. These resulting data frames were then labeled and saved to .csv files for further analysis.

Computing HRV Measures. Each of these data streams was processed using Kubios 2.2 (Tarvainen, Niskanen, Lipponen, Ranta-aho, \& Karjalainen, 2013), a software designed specifically for HRV analysis. Using Kubios, the following time and frequency parameters of cardiac function were calculated (among others) for the ECG as well as the E4 data:

1. Mean heart rate (meanhr)

2. SDNN

3. Total power (TP) 
4. Power in the low frequency range $(0.04-0.12 \mathrm{~Hz})(\mathrm{LFP})$

5. Power in the high frequency range $(0.12-0.4 \mathrm{~Hz})(\mathrm{HFP})$

Calculating Missingness. In order to assess the quality of the E4-derived HRV data, we created a metric called 'missingness'. As estimates of heart rate are generally robust and highly correlated across devices and across conditions, these can be used to estimate the number of IBIs that should have been recorded. Missingness was then calculated by dividing the observed IBIs by the expected number of heartbeats (meanhr * minutes). The observed IBIs were increased by 1 , so that it could be compared to heart rate.

$$
\text { missingness }=1-\frac{\text { observed IBIs }+1}{\text { Meanhr } * \text { minutes }}
$$

The data was then filtered based on the degree of missingness. We tested and compared thresholds for missingness. Data was filtered such that cases missing more than $35 \%$ and then more than $25 \%$ of the heartbeats were excluded from analysis. Sample R code used to implement this method can be found in Appendix A.

\section{Results}

We first matched session data to participants. After combining all data into a continuous table, data were filtered to the timeframe in which participants completed tasks. As the E4 record IBIs only when data quality is strong, in many cases participants had no recorded data. This was especially problematic in tasks that had a shorter duration or those involving movement. IBIs were recorded for 62 participants in the supine condition, 56 participants in the seated baseline condition, 50 in the standing condition, 25 in the non-dominant grip condition, and 20 in the dominant grip condition. 
Pearson's correlations were conducted comparing a range of HRV metrics obtained from the E4 wristbands, to those obtained from the Biopac devices (see Table 1). While mean heart rate was closely correlated in all conditions, the metrics of heart rate variability were not as strongly correlated as one would expect from devices assumed to be measuring the same thing. However, many files had large sequences where IBIs were not recorded due to poor signal. Using our novel method of error detection discussed above, we next tested to see if correlations between E4 and Biopac devices could be improved by excluding files with poor quality data. Note that the same pattern of results emerged when calculating Spearman's correlations, which use rank ordering and are, therefore, less susceptible than Pearson's correlations to artificially inflated estimates due to potentially influential data-points or extreme values.

Filtering cases resulted in loss of data but a large increase in the correlation between Biopac and E4 devices (see Tables 2 and 3). When missingness was less than .35, 15 cases were lost from the baseline condition but the correlation between E4 and Biopac was improved from .72 to .97 . Decreasing the threshold further from .35 to .25 removed an additional 10 cases in the baseline condition but the correlations were not greatly improved. In some conditions (grip and standing conditions), relatively few participants had any useable data at a cutoff of .35 . This resulted in poorer correlations in the data between the E4 wristbands and the Biopac device.

\section{Discussion}

Minimizing motion artifact is a major challenge to the development of equipment to reliably assess HRV and other indices of cardiovascular performance and health. Results of the present study indicate that despite being designed specifically for ambulatory use, the E4, like other devices for assessing HRV, still suffers from issues of motion artifact. Specifically, when 
data were filtered to exclude cases where $35 \%$ or more of the E4 heartbeat data were missing, the number of viable data points was reduced by almost half (or more) in all but the seated and supine resting conditions. Setting the cutoff at $25 \%$ missingness yielded even fewer viable cases across all conditions. In other words, the E4 performed best when participants were sitting or lying still. However, even in these conditions, correlations between frequency-derived HRV measures (HFP, LFP) assessed via the E4 and wired ECG were moderate to strong (.40 - .82). Excluding cases that were missing more than $35 \%$ and more than $25 \%$ of heartbeats increased these correlations to .86 to .97 and .85 to .98 , respectively, but greatly reduced the number of viable cases. When participants were standing or engaging in the typing task or grip task, data quality suffered greatly such that excluding cases with missingness greater than $25 \%$ depleted the number of viable cases to 15 or fewer, too few to produce reliable estimates of HRV.

The key contribution of this study is the technique for assessing error in HRV data collected with the E4 or other PPG-based wristbands, and for determining and implementing cutoff values for error tolerance. Estimating error directly, can help researchers to better evaluate the quality of their data and exclude affected data, thus minimizing the impact of error on results. In our sample, when error levels were adjusted to less than .25 , the correlation between E4 and wired-ECG derived measures of high frequency HRV for the standing condition increased from .20 to .90 . However, in doing so, data from more than half of the participants were excluded. When applying error tolerance cutoffs to the typing data, virtually all data were rejected. It remains to be seen whether other wristband devices of HRV measurement would do any better than the test case of E4 we used in this study.

Wristband measures are appealing for their non-obtrusive nature and comfort. But due to the frequent movement of hands and arms, other sites might be better than the wrist. Ambulatory 
measures that strap around the chest or clip to the earlobe are being developed and may minimize artifact. Given the problems with motion artifact, researchers are also focusing both on improving hardware and improving algorithms for cleaning and processing data, to minimize the influence of motion artifact. For example, efforts are being made to use accelerometers in conjunction with motion artifact reduction algorithms to improve data quality (e.g., Han, Kim, \& Kim, 2007; Han \& Kim, 2012; Kim, Ryoo, \& Bae, 2007; Yousefi, Nourani, Ostadabbas, \& Panahi, 2014). Other researchers are examining linear prediction analysis (LPC) and wavelet transformation techniques to reduce the impact of motion artifact and accurately estimate HRV (Alqaraawi et al, 2016). As those developments occur, the HRV data quality will likely improve. However, any new techniques or turnkey tools must be validated against the tried-and-tested ECG-based method of calculating HRV, as we did in this study. The algorithm for error detection and tolerance that we introduce in this paper is relatively simple to implement and can be easily adapted by researchers to evaluate the quality of HRV data obtained from a wide variety of ambulatory devices. We hope it will facilitate future research on ambulatory devices for measuring HRV.

\section{References}


Acharya, U. R., Joseph, K. P., Kannathal, N., Lim, C. M., \& Suri, J. S. (2006). Heart rate variability: a review. Medical and Biological Engineering and Computing, 44, 10311051.

Akselrod, S., Gordon, D., Ubel, F. A., Shannon, D. C., Barger, A. C., \& Cohen, R. J. (1981). Power spectrum analysis of heart rate fluctuation: a quantitative probe of beat-to-beat cardiovascular control. Science, 213, 220-222.

Akselrod, S., Eliash, S., Oz, O., \& Cohen, S. (1987). Hemodynamic regulation in SHR: investigation by spectral analysis. American Journal of Physiology-Heart and Circulatory Physiology, 253(1), H176-H183.

Allen, J. (2007). Photoplethysmography and its application in clinical physiological measurement. Physiological Measurement, 28(3), R1-R39.

Allen, J. J., Chambers, A. S., \& Towers, D. N. (2007). The many metrics of cardiac chronotropy: A pragmatic primer and a brief comparison of metrics. Biological Psychology, 74(2), 243-262.

Alqaraawi, A., Alwosheel, A., \& Alasaad, A. (2016, May). Towards efficient heart rate variability estimation in artifact-induced Photoplethysmography signals. In Electrical and Computer Engineering (CCECE), 2016 IEEE Canadian Conference on (pp. 1-6). IEEE.

Appelhans, B. M., \& Luecken, L. J. (2006). Heart rate variability as an index of regulated emotional responding. Review of General Psychology, 10(3), 229-240.

Arberet, S., Lemay, M., Renevey, P., Sola, J., Grossenbacher, O., Andries, D., ... \& Bertschi, M. (2013, September). Photoplethysmography-based ambulatory heartbeat monitoring embedded into a dedicated bracelet. In Computing in Cardiology Conference (CinC), 2013 (pp. 935-938). IEEE. 
Berntson, G. G., Cacioppo, J. T., \& Quigley, K. S. (1991). Autonomic determinism: the modes of autonomic control, the doctrine of autonomic space, and the laws of autonomic constraint. Psychological review, 98, 459-487.

Berntson, G. G., Bigger, J. T., Eckberg, D. L., Grossman, P., Kaufmann, P. G., Malik, M., ... \& van der Mollen, M. W. (1997). Heart rate variability: origins, methods, and interpretive caveats. Psychophysiology, 34, 623-648.

Bertsch, K., Hagemann, D., Naumann, E., Schächinger, H., \& Schulz, A. (2012). Stability of heart rate variability indices reflecting parasympathetic activity. Psychophysiology, 49, $672-682$.

Bolanos, M., Nazeran, H., \& Haltiwanger, E. (2006, August). Comparison of heart rate variability signal features derived from electrocardiography and photoplethysmography in healthy individuals. In Engineering in Medicine and Biology Society, 2006. EMBS'06. 28th Annual International Conference of the IEEE (pp. 4289-4294). IEEE.

Calkins, S. D., \& Johnson, M. C. (1998). Toddler regulation of distress to frustrating events: Temperamental and maternal correlates. Infant Behavior and Development, 21, 379-395.

Carney, R. M., Blumenthal, J. A., Stein, P. K., Watkins, L., Catellier, D., Berkman, L. F., ... \& Freedland, K. E. (2001). Depression, heart rate variability, and acute myocardial infarction. Circulation, 104(17), 2024-2028.

Challoner, A. V. J. (1979). Photoelectric plethysmography for estimating cutaneous blood flow. Non-invasive Physiological Measurements, 1, 125-151.

Chambers, A. S., \& Allen, J. J. (2002). Vagal tone as an indicator of treatment response in major depression. Psychophysiology, 39, 861-864. 
Corino, V. D. A., Matteucci, M., \& Mainardi, L. T. (2007). Analysis of heart rate variability to predict patient age in a healthy population. Methods of Information in Medicine, 46(2), 191-195.

Davy, K. P., Desouza, C. A., Jones, P. P., \& Seals, D. R. (1998). Elevated heart rate variability in physically active young and older adult women. Clinical Science, 94(6), 579-584.

Drinnan, M., Allen, J., Langley, P., \& Murray, A. (2000). Detection of sleep apnoea from frequency analysis of heart rate variability. In Computers in Cardiology 2000 (pp. 259262). IEEE.

Einthoven, W., Fahr, G., \& De Waart, A. (1913). Über die Richtung und die manifeste Grösse der Potentialschwankungen im menschlichen Herzen und über den Einfluss der Herzlage auf die Form des Elektrokardiogramms. Pflügers Archiv European Journal of Physiology, 150(6), 275-315.

Elsenbruch, S., Harnish, M. J., \& Orr, W. C. (1999). Heart rate variability during waking and sleep in healthy males and females. Sleep, 22(8), 1067-1071.

Ewing, D. J., Borsey, D. Q., Bellavere, F., \& Clarke, B. F. (1981). Cardiac autonomic neuropathy in diabetes: comparison of measures of RR interval variation. Diabetologia, 21(1), 18-24.

Fabes, R. A., \& Eisenberg, N. (1997). Regulatory control and adults' stress-related responses to daily life events. Journal of Personality and Social Psychology, 73, 1107-1117.

Foo, J. Y. A., \& Wilson, S. J. (2006). A computational system to optimise noise rejection in photoplethysmography signals during motion or poor perfusion states. Medical and Biological Engineering and Computing, 44, 140-145. 
Friedman, B. H. (2007). An autonomic flexibility-neurovisceral integration model of anxiety and cardiac vagal tone. Biological Psychology, 74, 185-199.

Garbarino, M., Lai, M., Bender, D., Picard, R. W., \& Tognetti, S. (2014, November). Empatica E3-A wearable wireless multi-sensor device for real-time computerized biofeedback and data acquisition. In Wireless Mobile Communication and Healthcare (Mobihealth), 2014 EAI 4th International Conference on (pp. 39-42). IEEE.

Ge, D., Srinivasan, N., \& Krishnan, S. M. (2002). Cardiac arrhythmia classification using autoregressive modeling. Biomedical Engineering Online, 1(1), 5-17.

Geisler, F. C., Kubiak, T., Siewert, K., \& Weber, H. (2013). Cardiac vagal tone is associated with social engagement and self-regulation. Biological Psychology, 93, 279-286.

Gil, E., Orini, M., Bailón, R., Vergara, J. M., Mainardi, L., \& Laguna, P. (2010, August). Timevarying spectral analysis for comparison of HRV and PPG variability during tilt table test. In Engineering in Medicine and Biology Society (EMBC), 2010 Annual International Conference of the IEEE (pp. 3579-3582). IEEE.

Goldstein, D. S., Bentho, O., Park, M. Y., \& Sharabi, Y. (2011). Low-frequency power of heart rate variability is not a measure of cardiac sympathetic tone but may be a measure of modulation of cardiac autonomic outflows by baroreflexes. Experimental Physiology, 96, $1255-1261$.

Grossmann, I., Sahdra, B. K., \& Ciarrochi, J. (2016). A Heart and a mind: Self-distancing facilitates the association between heart rate variability and wise reasoning. Frontiers in Behavioral Neuroscience, 10:68. DOI: 10.3389/fnbeh.2016.00068. 
Grossman, P., \& Taylor, E. W. (2007). Toward understanding respiratory sinus arrhythmia: relations to cardiac vagal tone, evolution and biobehavioral functions. Biological Psychology, 74, 263-285.

Guzzetti, S., Piccaluga, E., Casati, R., Cerutti, S., Lombardi, F., Pagani, M., \& Malliani, A. (1988). Sympathetic predominance an essential hypertension: a study employing spectral analysis of heart rate variability. Journal of Hypertension, 6, 711-717.

Han, H., \& Kim, J. (2012). Artifacts in wearable photoplethysmographs during daily life motions and their reduction with least mean square based active noise cancellation method. Computers in Biology and Medicine, 42, 387-393.

Han, H., Kim, M. J., \& Kim, J. (2007, August). Development of real-time motion artifact reduction algorithm for a wearable photoplethysmography. In Engineering in Medicine and Biology Society, 2007. EMBS 2007. 29th Annual International Conference of the IEEE (pp. 1538-1541). IEEE.

Jabaudon, D., Sztajzel, J., Sievert, K., Landis, T., \& Sztajzel, R. (2004). Usefulness of ambulatory 7-day ECG monitoring for the detection of atrial fibrillation and flutter after acute stroke and transient ischemic attack. Stroke, 35, 1647-1651.

Jago, J. R., \& Murray, A. (1988). Repeatability of peripheral pulse measurements on ears, fingers and toes using photoelectric plethysmography. Clinical Physics and Physiological Measurement, 9, 319-330.

Jeyhani, V., Mahdiani, S., Peltokangas, M., \& Vehkaoja, A. (2015, August). Comparison of HRV parameters derived from photoplethysmography and electrocardiography signals. In Engineering in Medicine and Biology Society (EMBC), 2015 37th Annual International Conference of the IEEE (pp. 5952-5955). IEEE. 
Julu, P. O. O., Cooper, V. L., Hansen, S., \& Hainsworth, R. (2003). Cardiovascular regulation in the period preceding vasovagal syncope in conscious humans. The Journal of Physiology, 549(1), 299-311.

Kamal, A. A. R., Harness, J. B., Irving, G., \& Mearns, A. J. (1989). Skin photoplethysmography - a review. Computer Methods and Programs in Biomedicine, 28(4), 257-269.

Kamath, M. V., \& Fallen, E. L. (1995). Correction of the heart rate variability signal for ectopics and missing beats, In: M. Malik \& A. J. Camm (Eds.), Heart Rate Variability, (pp. $75-$ 85). Armonk, NY: Futura Pub. Co. Inc.

Katona, P. G., \& Jih, F. (1975). Respiratory sinus arrhythmia: noninvasive measure of parasympathetic cardiac control. Journal of Applied Physiology, 39, 801-805.

Kim, S. H., Ryoo, D. W., \& Bae, C. (2007, August). Adaptive noise cancellation using accelerometers for the PPG signal from forehead. In Engineering in Medicine and Biology Society, 2007. EMBS 2007. 29th Annual International Conference of the IEEE (pp. 2564-2567). IEEE.

Kleiger, R. E., Bigger, J. T., Bosner, M. S., Chung, M. K., Cook, J. R., Rolnitzky, L. M., ... \& Fleiss, J. L. (1991). Stability over time of variables measuring heart rate variability in normal subjects. The American journal of cardiology, 68, 626-630.

Kok, B. E., \& Fredrickson, B. L. (2010). Upward spirals of the heart: Autonomic flexibility, as indexed by vagal tone, reciprocally and prospectively predicts positive emotions and social connectedness. Biological psychology, 85, 432-436. 
Koudstaal, P. J., van Gijn, J., Klootwijk, A. P. J., Van Der Meche, F. G. A., \& Kappelle, L. J. (1986). Holter monitoring in patients with transient and focal ischemic attacks of the brain. Stroke, 17(2), 192-195.

Lu, G., \& Yang, F. (2009). Limitations of oximetry to measure heart rate variability measures. Cardiovascular Engineering, 9(3), 119-125.

Lu, G., Yang, F., Taylor, J. A., \& Stein, J. F. (2009). A comparison of photoplethysmography and ECG recording to analyse heart rate variability in healthy subjects. Journal of Medical Engineering \& Technology, 33, 634-641.

Malpas, S. C., Whiteside, E. A., \& Maling, T. J. (1991). Heart rate variability and cardiac autonomic function in men with chronic alcohol dependence. Heart, 65(2), 84-88.

Masi, C. M., Hawkley, L. C., Rickett, E. M., \& Cacioppo, J. T. (2007). Respiratory sinus arrhythmia and diseases of aging: Obesity, diabetes mellitus, and hypertension. Biological Psychology, 74, 212-223.

McCarthy, C., Pradhan, N., Redpath, C., \& Adler, A. (2016). Validation of the Empatica E4 wristband. In Student Conference (ISC), 2016 IEEE EMBS International, pp. 1-4.

Miu, A. C., Heilman, R. M., \& Miclea, M. (2009). Reduced heart rate variability and vagal tone in anxiety: trait versus state, and the effects of autogenic training. Autonomic Neuroscience, 145(1), 99-103.

Muaremi, A., Arnrich, B., \& Tröster, G. (2013). Towards measuring stress with smartphones and wearable devices during workday and sleep. BioNanoScience, 3(2), 172-183.

Murray, A., Ewing, D. J., Campbell, I. W., Neilson, J. M., \& Clarke, B. F. (1975). RR interval variations in young male diabetics. Heart, 37, 882-885. 
Ollander, S., Godin, C., Campagne, A., \& Charbonnier, S. (2016, October). A comparison of wearable and stationary sensors for stress detection. In Systems, Man, and Cybernetics (SMC), 2016 IEEE International Conference on (pp. 004362-004366). IEEE.

Pagani, M., Montano, N., Porta, A., Malliani, A., Abboud, F. M., Birkett, C., \& Somers, V. K. (1997). Relationship between spectral components of cardiovascular variabilities and direct measures of muscle sympathetic nerve activity in humans. Circulation, 95, 14411448.

Pagani, M., Lombardi, F., Guzzetti, S., Rimoldi, O., Furlan, R., Pizzinelli, P., ... \& Malliani, A. (1986). Power spectral analysis of heart rate and arterial pressure variabilities as a marker of sympatho-vagal interaction in man and conscious dog. Circulation Research, 59(2), 178-193.

Paradiso, R., Faetti, T., \& Werner, S. (2011, August). Wearable monitoring systems for psychological and physiological state assessment in a naturalistic environment. In Engineering in Medicine and Biology Society, EMBC, 2011 Annual International Conference of the IEEE (pp. 2250-2253). IEEE.

Pope 3rd, C. A., Eatough, D. J., Gold, D. R., Pang, Y., Nielsen, K. R., Nath, P., ... \& Kanner, R. E. (2001). Acute exposure to environmental tobacco smoke and heart rate variability. Environmental Health Perspectives, 109, 711-716.

Porges, S. W. (1992). Vagal tone: a physiologic marker of stress vulnerability. Pediatrics, 90(3), 498-504.

Porges, S. W. (2007). The polyvagal perspective. Biological Psychology, 74(2), 116-143. 
Preejith, S. P., Alex, A., Joseph, J., \& Sivaprakasam, M. (2016, May). Design, development and clinical validation of a wrist-based optical heart rate monitor. In Medical Measurements and Applications (MeMeA), 2016 IEEE International Symposium on(pp. 1-6). IEEE.

R Core Team. (2017). R: A language and environment for statistical computing. R Foundation for Statistical Computing, Vienna, Austria. URL http://www.R-project.org.

Rauh, R., Limley, R., Bauer, R. D., Radespiel-Troger, M., \& Mueck-Weymann, M. (2004, August). Comparison of heart rate variability and pulse rate variability detected with photoplethysmography. In Saratov Fall Meeting 2003: Optical Technologies in Biophysics and Medicine V (pp. 115-126). International Society for Optics and Photonics.

Renevey, P., Vetter, R., Krauss, J., Celka, P., \& Depeursinge, Y. (2001). Wrist-located pulse detection using IR signals, activity and nonlinear artifact cancellation. In Engineering in Medicine and Biology Society, 2001. Proceedings of the 23rd Annual International Conference of the IEEE (Vol. 3, pp. 3030-3033). IEEE.

Romero, I., Berset, T., Buxi, D., Brown, L., Penders, J., Kim, S., ... \& Yazicioglu, F. (2011, October). Motion artifact reduction in ambulatory ECG monitoring: an integrated system approach. In Proceedings of the 2nd Conference on Wireless Health (p. 11). ACM.

Rottenberg, J. (2007). Cardiac vagal control in depression: a critical analysis. Biological Psychology, 74(2), 200-211.

Sahdra, B. K., Ciarrochi, J., \& Parker, P. D. (2015). High-frequency heart rate variability linked to affiliation with a new group. PLoS ONE, 10(6), e 0129583.

DOI:10.1371/journal.pone.0129583. 
Sandercock, G. R. H., Shelton, C., Bromley, P., \& Brodie, D. A. (2004). Agreement between three commercially available instruments for measuring short-term heart rate variability. Physiological Measurement, 25, 1115-1124.

Saul, J. P., Albrecht, P., Berger, R. D., \& Cohen, R. J. (1987). Analysis of long term heart rate variability: methods, 1/f scaling and implications. Computers in cardiology, 14, 419-422.

Saul, J. P. (1990). Beat-to-beat variations of heart rate reflect modulation of cardiac autonomic outflow. Physiology, 5, 32-37.

Schäfer, A., \& Vagedes, J. (2013). How accurate is pulse rate variability as an estimate of heart rate variability?: A review on studies comparing photoplethysmographic technology with an electrocardiogram. International Journal of Cardiology, 166, 15-29.

Sherwood, A., Allen, M. T., Fahrenberg, J., Kelsey, R. M., Lovallo, W. R., \& van Dooren, L.J.P. (1990). Methodological guidelines for impedance cardiography. Psychophysiology, 27, 123.

Stein, P. K., \& Kleiger, R. E. (1999). Insights from the study of heart rate variability. Annual Review of Medicine, 50, 249-261.

Tamura, T., Maeda, Y., Sekine, M., \& Yoshida, M. (2014). Wearable photoplethysmographic sensors_-past and present. Electronics, 3, 282-302.

Tarvainen, M. P., Niskanen, J. P., Lipponen, J. A., Ranta-Aho, P. O., \& Karjalainen, P. A. (2014). Kubios HRV-heart rate variability analysis software. Computer Methods and Programs in Biomedicine, 113(1), 210-220.

Task Force of the European Society of Cardiology and the North American Society for Pacing and Electrophysiology (1996). Heart rate variability: standards of measurement, physiological interpretation, and clinical use. Circulation, 93, 1043-1065. 
Thayer, J., Hansen, A., Saus-Rose, E., \& Johnson, B. H. (2009). Heart rate variability, prefrontal neural function, and cognitive performance: The neurovisceral integration perspective on self-regulation, adaptation, and health. Annals of Behavioral Medicine Publication of the Society of Behavioral Medicine, 37(2), 141-153.

Thayer, J. F., \& Lane, R. D. (2007). The role of vagal function in the risk for cardiovascular disease and mortality. Biological Psychology, 74, 224-242.

Yousefi, R., Nourani, M., Ostadabbas, S., \& Panahi, I. (2014). A motion-tolerant adaptive algorithm for wearable photoplethysmographic biosensors. IEEE Journal of Biomedical and Health Informatics, 18, 670-681. 
Running Head: A method for evaluating PPG-based HRV devices

Table 1

Pearson's correlations between data obtained from E4 and Biopac.

\begin{tabular}{lcccccc}
\hline & meanhr & sdnn & HFP & LFP & TP & N \\
\hline Seated baseline & $.949^{*}$ & $.768^{*}$ & $.72^{*}$ & $.656^{*}$ & $.835^{*}$ & 56 \\
Dominant grip & $.977^{*}$ & $.714^{*}$ & $.714^{*}$ & $.602^{*}$ & $.769^{*}$ & 20 \\
Supine baseline & $.988^{*}$ & $.839^{*}$ & $.817^{*}$ & $.400^{*}$ & $.760^{*}$ & 62 \\
Non-dominant grip & $.967^{*}$ & $.877^{*}$ & $.761^{*}$ & .316 & $.671^{*}$ & 25 \\
Standing baseline & $.825^{*}$ & $.567^{*}$ & .204 & $.633^{*}$ & $.404^{*}$ & 50 \\
Typing & $.938^{*}$ & $.337^{*}$ & $.642^{*}$ & $.329 *$ & $.54 *$ & 40 \\
\hline
\end{tabular}

Note. meanhr $=$ mean heart rate; $\mathrm{HFP}=$ high frequency power; $\mathrm{LFP}=$ low frequency power; $\mathrm{TP}=$ total power; $\mathrm{N}=$ number of participants with data.

$* p<.05$ 
Running Head: A method for evaluating PPG-based HRV devices

Table 2

Pearson's correlations between data obtained from E4 and Biopac. Missingness $<$ .35

\begin{tabular}{lcccccc}
\hline & meanhr & sdnn & HFP & LFP & TP & N \\
\hline Seated baseline & $.992^{*}$ & $.948^{*}$ & $.970^{*}$ & $.880^{*}$ & $.976^{*}$ & 41 \\
Dominant grip & $.986^{*}$ & $.861^{*}$ & $.911^{*}$ & $.815^{*}$ & $.923^{*}$ & 12 \\
Supine baseline & $.987^{*}$ & $.924^{*}$ & $.859^{*}$ & $0.879 *$ & $.862^{*}$ & 52 \\
Non-dominant grip & $.911^{*}$ & $.908^{*}$ & $.720^{*}$ & $0.597^{*}$ & $.778^{*}$ & 14 \\
Standing baseline & $.997^{*}$ & $.928^{*}$ & $.898^{*}$ & $0.951^{*}$ & $.945^{*}$ & 20 \\
Typing & - & - & - & - & & 2 \\
\hline
\end{tabular}

Note. meanhr $=$ mean heart rate; $\mathrm{HFP}=$ high frequency power; $\mathrm{LFP}=$ low frequency power; $\mathrm{TP}=$ total power; $\mathrm{N}=$ number of participants with data.

$* p<.05$. 
Running Head: A method for evaluating PPG-based HRV devices

Table 3

Pearson's correlations between data obtained from E4 and Biopac. Missingness < .25

\begin{tabular}{lcccccc}
\hline & meanhr & sdnn & HFP & LFP & TP & N \\
\hline Seated baseline & $.991^{*}$ & $.954^{*}$ & $.976^{*}$ & $.883^{*}$ & $.973^{*}$ & 31 \\
Dominant grip & $.998^{*}$ & $.944^{*}$ & .615 & -.225 & .415 & 6 \\
Supine baseline & $.994^{*}$ & $.918^{*}$ & $.850^{*}$ & $.873^{*}$ & $.854^{*}$ & 48 \\
Non-dominant grip & $.933^{*}$ & $.813^{*}$ & .519 & .377 & .242 & 9 \\
Standing baseline & $.999^{*}$ & $.954^{*}$ & $.917 *$ & $.960 *$ & $.954^{*}$ & 15 \\
Typing & - & - & - & - & - & 1 \\
\hline
\end{tabular}

Note. meanhr $=$ mean heart rate; $\mathrm{HFP}=$ high frequency power; $\mathrm{LFP}=$ low frequency power; $\mathrm{TP}=$ total power; $\mathrm{N}=$ number of participants with data.

$* p<.05$. 


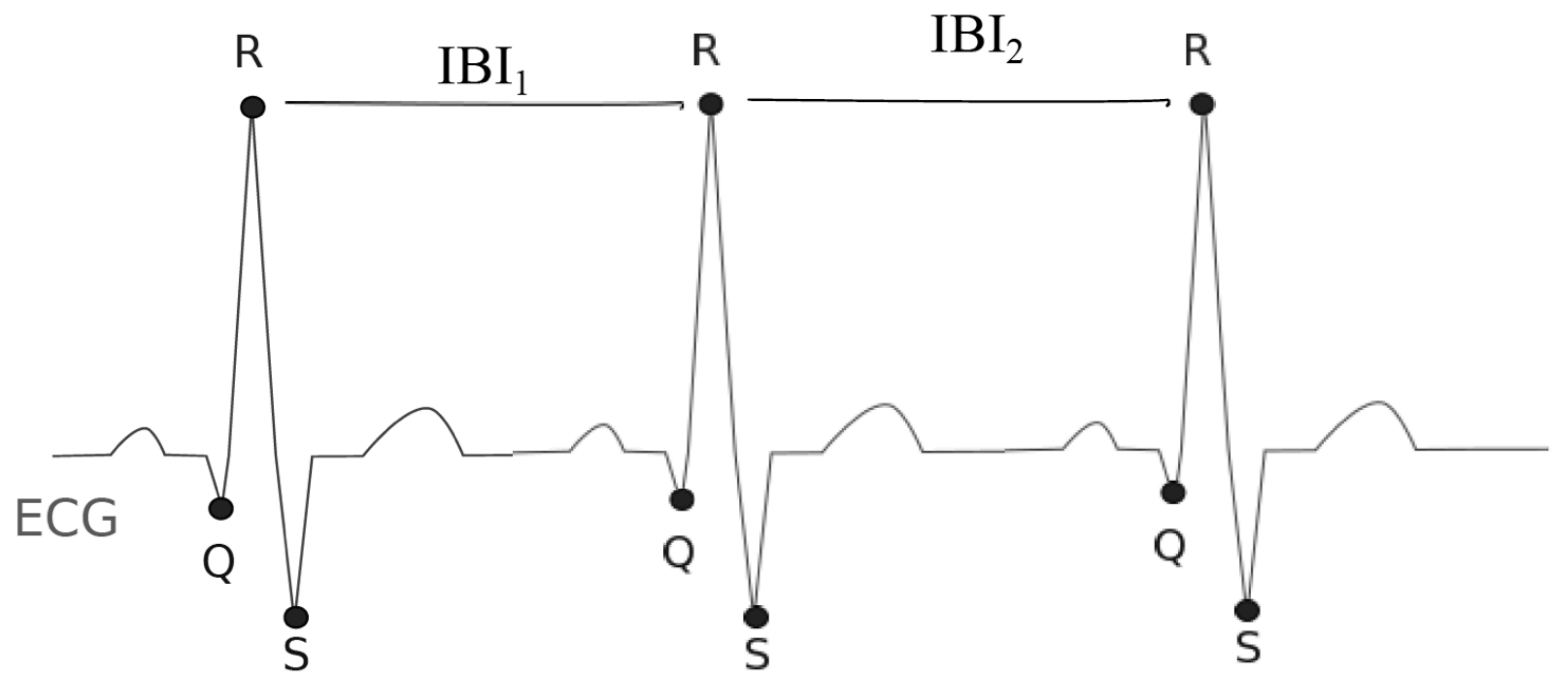

Figure 1. Schematic diagram of ECG waveform with IBI or R-R intervals. 